\title{
Broadband Light Harvesting Nanostructures Robust to Edge Bluntness
}

\author{
Yu Luo, * Dang Yuan Lei, Stefan A. Maier, and J. B. Pendry \\ The Blackett Laboratory, Department of Physics, Imperial College London, \\ London SW7 2AZ, United Kingdom
}

(Received 12 October 2011; published 11 January 2012)

\begin{abstract}
Metallic structures with sharp corners harvest the energy of incident light through plasmonic resonances, concentrating it in the corners and greatly increasing the local energy density. Despite its wide array of applications, this effect is normally strongly dependent on how sharp the corners are, presenting problems for fabrication. In this Letter, an analytical approach is proposed, based on transformation optics, to investigate a general class of plasmonic nanostructures with blunt edges or corners. Comprehensive discussions are provided on how the geometry affects the local field enhancement as well as the frequency and energy of each plasmonic resonance. Remarkably, our results evidence the possibility of designing broadband light harvesting devices with an absorption property insensitive to the geometry bluntness.
\end{abstract}

Surface plasmons (SPs) are coherent electron oscillations that offer subwavelength light confinement at a metal-dielectric interface [1-4]. A metallic nanoparticle of finite size usually sustains SP resonances at quantized frequencies [1,2], but recent theoretical work has shown that this no longer holds true for nanostructures with singularities such as a sharp edge or corner $[5,6]$. Transformation optics [7] can explain this counterintuitive phenomenon, which shows that the SP spectrum of a singular nanoparticle is identical to that of an infinite metallodielectric system and is therefore continuous rather than discrete. The SP modes supported by a singular plasmonic structure (such as the crescent $[5,6]$ or kissing cylinders [8]) propagate towards the singularity, slowing down as they progress and never reaching the singularity. Therefore, an extremely large field enhancement and a continuous absorption cross section are expected over a broad frequency band.

However, considering experimental realizations, singularities or perfectly sharp boundaries in those structures are unlikely due to limitations in fabrication techniques and the surface tension of the metal. In real-world applications, plasmonic nanostructures always contain blunt tips [9-18]. Therefore, the possibility of quantitatively examining how the edge rounding at the sharp boundary will alter the optical responses (particularly, the field enhancement and light harvesting property) has great significance on both theoretical and practical levels. While many nanostructures with blunt edges have been specifically investigated with numerical [12-14] or experimental methods [9-11,15-18], there has never been a systematic strategy reported so far to analytically deal with this problem. In this Letter, we propose an analytical model for a general class of blunt plasmonic devices by applying conformal mappings to the truncated metallodielectric system associated with the singular structures. To illustrate the usefulness of this approach, we consider one of the most commonly used singular structures, the two-dimensional (2D) nanocrescent, and study how the energy and bandwidth of each plasmonic resonance can be tailored by tuning the geometry. The analytical treatment reveals that the resonant properties of each SP mode are directly related to the crescent thickness $t$ and the vertex angle $\theta$ formed at the crescent tip [ $t$ and $\theta$ are defined in Fig. 1(b)]. Interestingly, by appropriately adjusting these parameters, a relatively broadband absorption cross section nearly independent of the tip bluntness can be obtained. This property is beneficial for experimental realizations, since it relaxes the requirements on the fabrication precision at sharp tips. With regard to the local energy density, although the bluntness causes a remarkable reduction of the maximum field
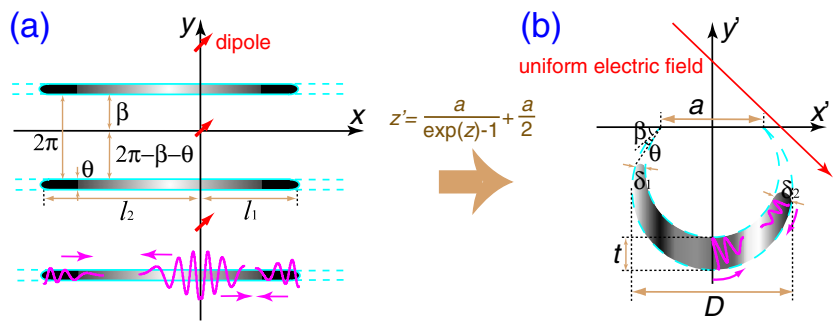

FIG. 1 (color online). The coordinate transformation which bridges (a) the truncated periodic metallodielectric system and (b) the blunt crescent. Here, $z=x+i y$ and $z^{\prime}=x^{\prime}+i y^{\prime}$ are usual complex numbers, $D$ is the total dimension of the crescent, $a$ denotes the distance between the two crescent tips, $t$ represents the maximum distance between the inner and outer crescent boundaries (referred to as the crescent thickness), $\theta$ is the angle formed at the crescent tips, and $\beta$ denotes the angle between the $x$ axis and the outer boundary of the crescent. 
enhancement at the geometry tips, as predictable by intuition, the field distribution at the rest part of the crescent is almost unaffected. The proposed strategy is not restricted to the specific structure we discuss here. By adopting different transformations, it could be used to treat a general class of 2D blunt nanostructures and even be extended to three-dimensional (3D) cases, thereby providing a powerful tool for the design of practical plasmonic devices.

Figures 1(a) and 1(b) show the basic idea of our transformation strategy and the general structure under investigation. In our recent study, an original approach based on transformation optics has been proposed to study the optical responses of a series of singular plasmonic structures $[5,6]$. For a singular structure that is difficult to analyze with traditional electromagnetic methods, one could find a shortcut by transforming it into another structure on which the analytical solution may be easily found. For instance, under a conformal mapping $z^{\prime}=a /\left(e^{z}-1\right)+a / 2$ (with the parameters $a, z$, and $z^{\prime}$ defined in the figure caption of Fig. 1), which links periodic infinitely long metallic slabs [shown by dashed lines in Fig. 1(a)] and a crescent-shaped cylinder with perfectly sharp tips [shown by dashed lines in Fig. 1(b)], the absorption properties of the structures are preserved. Thus, the singular crescent exhibits a continuous absorption cross section over a broadband spectrum, similar to the infinite metallodielectric plasmonic ensemble. Now, we modify our strategy to study the blunt nanocrescent, which is simply obtained by removing the perfectly sharp tips of the singular crescent structure [see the shaded (dark) region in Fig. 1(b)]. Accordingly, in the initial space (the $x-y$ frame), the infinite metallodielectric system is truncated on both sides [see the shaded (dark) region in Fig. 1(a)]. The distances from the dipole to the two truncation points $l_{1}$ and $l_{2}$ determine, respectively, the bluntness of each tip of the crescent:

$\delta_{1(2)}=\frac{D \sin \beta \tan (\theta / 2)}{\left|\sinh \left[\left(l_{1(2)}+i \beta\right) / 2\right]\right|\left|\sinh \left[\left(l_{1(2)}+i \beta+i \theta\right) / 2\right]\right|}$.

Here, $\delta_{1}$ and $\delta_{2}$ denote the bluntness dimensions (or the diameters at the two blunt tips). The remaining geometrical parameters $D, \theta$, and $\beta$ are all defined in the figure caption of Fig. 1.

Under the condition that the dimension of the crescent is sufficiently small (typically $D \leq 40 \mathrm{~nm}$ ), the near-field approximation can be applied in our derivation. The dipole arrays in the original space are transformed into a uniform electric field $E_{0}^{\prime}$, which enables a good approximation of an incident plane wave. In this case, the material properties of the nanostructure and the electrostatic potential are preserved under conformal mappings [5,6]. Therefore, we can study analytically the resonant behavior of the blunt nanocrescent under a plane wave illumination by solving the problem of periodic truncated slabs in the quasistatic limit
[19]. Since the whole system under investigation has a finite physical size, the SP modes are now distributed at several discrete frequencies, with the resonant condition given by

$$
\begin{aligned}
& {\left[\frac{\varepsilon_{s}(\omega)-1}{\varepsilon_{s}(\omega)+1}\right]^{2}\left[e^{n\left[(2 \pi-\theta) /\left(\tau_{1}+\tau_{2}\right)\right]}-e^{n\left[\theta /\left(\tau_{1}+\tau_{2}\right)\right]}\right]^{2}} \\
& \quad-\left[e^{n\left[2 \pi /\left(\tau_{1}+\tau_{2}\right)\right]}-1\right]^{2}=0,
\end{aligned}
$$

where $\tau_{1(2)}=l_{1(2)} / \pi, n$ is the angular moment of the SP modes, and $\varepsilon_{s}(\omega)$ is the permittivity of silver at the frequency $\omega$.

We first examine how the geometry bluntness affects the number of SP modes and the distribution of these modes in the frequency domain. Figure 2(a) depicts the number of SP modes supported by four blunt silver crescents of different shapes. The material property of silver is taken from the experimental data [20] in all the derivations. Generally, the number of SP modes decreases exponentially as the tip bluntness increases. From Fig. 2(a), we can also see that altering the crescent thickness $t$ only leads to a slight shift of the curve and increasing the tip angle $\theta$ results in a smaller slope of the curve. In other words, SP modes on the crescent with a large tip angle are relatively robust to the geometry bluntness. As we will show, this fact provides a potential possibility for optimizing the absorption behavior of the blunt structure; Fig. 2(b) displays the resonant frequencies of a specific silver blunt crescent and the SP dispersion of the corresponding singular structure (with the same $\theta$ and $t$ ). The black curve stands for the case of a singular crescent where a continuous spectrum of interactions with light is expected, and the red circles correspond to the quantized modes exhibited by the blunt structure. It is worth noting that these modes are equally spaced in the $k$ space, indicating a none-equal distribution in the frequency domain, and the high-order resonances are compressed towards the surface plasmon frequency $\omega_{\mathrm{sp}}$. (a)

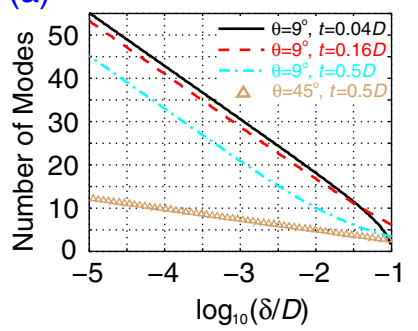

(b) $\times 10^{14}$

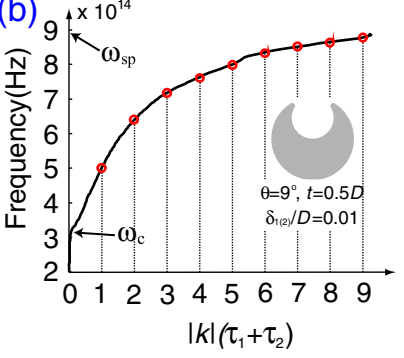

FIG. 2 (color online). (a) Number of SP modes as a function of the tip bluntness. Here, four crescents with different $\theta$ and $t$ (shown in the inset) are considered. In all the cases, we assume that the two tips of the crescent are equally blunt $\left(\delta_{1}=\delta_{2}\right)$. (b) The resonant features of a silver blunt crescent $\left(\theta=9^{\circ}\right.$, $t=0.5 D$, and $\delta_{1}=\delta_{2}=0.01 D$ ) and the corresponding singular structure. 
We next study the effect of the crescent shape on the extinction properties of SP modes, and this discussion finally leads to a blunt plasmonic structure for broadband light harvesting. In the quasistatic limit, the extinction cross section $\sigma_{\text {ext }}$ and the scattering cross section $\sigma_{\text {sca }}$ of the crescent can be directly deduced from the power dissipated by the dipole in the original $x-y$ frame:

$$
\begin{aligned}
& \sigma_{\text {ext }}^{x}=-4 k_{0} \operatorname{Im}\left\{\frac{\xi_{x x}}{1+i \eta \xi_{x x}}\right\}\left(\frac{D \sin \theta}{\tau_{1}+\tau_{2}}\right)^{2}, \\
& \sigma_{\text {ext }}^{y}=-4 k_{0} \operatorname{Im}\left\{\frac{\xi_{y y}}{1+i \eta \xi_{y y}}\right\}\left(\frac{D \sin \theta}{\tau_{1}+\tau_{2}}\right)^{2},
\end{aligned}
$$

$$
\begin{gathered}
\sigma_{\text {sca }}^{x}=2 k_{0}^{3}\left|\frac{\xi_{x x}}{1+i \eta \xi_{x x}}\right|^{2}\left(\frac{D \sin \theta}{\tau_{1}+\tau_{2}}\right)^{4}, \\
\sigma_{\text {sca }}^{y}=2 k_{0}^{3}\left|\frac{\xi_{y y}}{1+i \eta \xi_{y y}}\right|^{2}\left(\frac{D \sin \theta}{\tau_{1}+\tau_{2}}\right)^{4},
\end{gathered}
$$

where $k_{0}=\omega \sqrt{\varepsilon_{0} \mu_{0}}$ is the wave number in free space and the coefficients $\xi_{x x}, \xi_{y y}$, and $\eta$ take the following forms:

$$
\begin{aligned}
\xi_{x x} & =\sum_{n} \frac{n \pi}{2}\left(1-\cos \frac{2 n \pi \tau_{1}}{\tau_{1}+\tau_{2}}\right) \frac{e^{\alpha}\left(\gamma_{3}^{2 n}-1\right)\left(\gamma_{1}^{2 n}+\gamma_{2}^{2 n}\right)-2 e^{2 \alpha} \gamma_{3}^{2 n}+2\left(e^{2 \alpha}-1\right) \gamma_{1}^{n} \gamma_{2}^{n} \gamma_{3}^{n}+2}{e^{2 \alpha}\left(\gamma_{1}^{n} \gamma_{2}^{n}-\gamma_{3}^{n}\right)^{2}-\left(\gamma_{1}^{n} \gamma_{2}^{n} \gamma_{3}^{n}-1\right)^{2}} \\
\xi_{y y} & =\sum_{n} \frac{n \pi}{2}\left(1+\cos \frac{2 n \pi s_{1}}{s_{1}+s_{2}}\right) \frac{e^{\alpha}\left(\gamma_{3}^{2 n}-1\right)\left(\gamma_{1}^{2 n}+\gamma_{2}^{2 n}\right)+2 e^{2 \alpha} \gamma_{3}^{2 n}-2\left(e^{2 \alpha}-1\right) \gamma_{1}^{n} \gamma_{2}^{n} \gamma_{3}^{n}-2}{e^{2 \alpha}\left(\gamma_{1}^{n} \gamma_{2}^{n}-\gamma_{3}^{n}\right)^{2}-\left(\gamma_{1}^{n} \gamma_{2}^{n} \gamma_{3}^{n}-1\right)^{2}} \\
\eta & =\frac{1}{2}\left(\frac{\pi k_{0} D \sin \theta}{l_{1}+l_{2}}\right)^{2}
\end{aligned}
$$

where $\alpha=\ln \left[\left(\varepsilon_{s}-1\right) /\left(\varepsilon_{s}+1\right)\right], \gamma_{1}=e^{\beta /\left(\tau_{1}+\tau_{2}\right)}, \gamma_{2}=$ $e^{(2 \pi-\theta-\beta) /\left(\tau_{1}+\tau_{2}\right)}$, and $\gamma_{3}=e^{\theta /\left(\tau_{1}+\tau_{2}\right)}$. The absorption cross section is then deduced as $\sigma_{\text {abs }}^{x, y}=\sigma_{\text {ext }}^{x, y}-\sigma_{\text {sca. }}^{x, y}$.

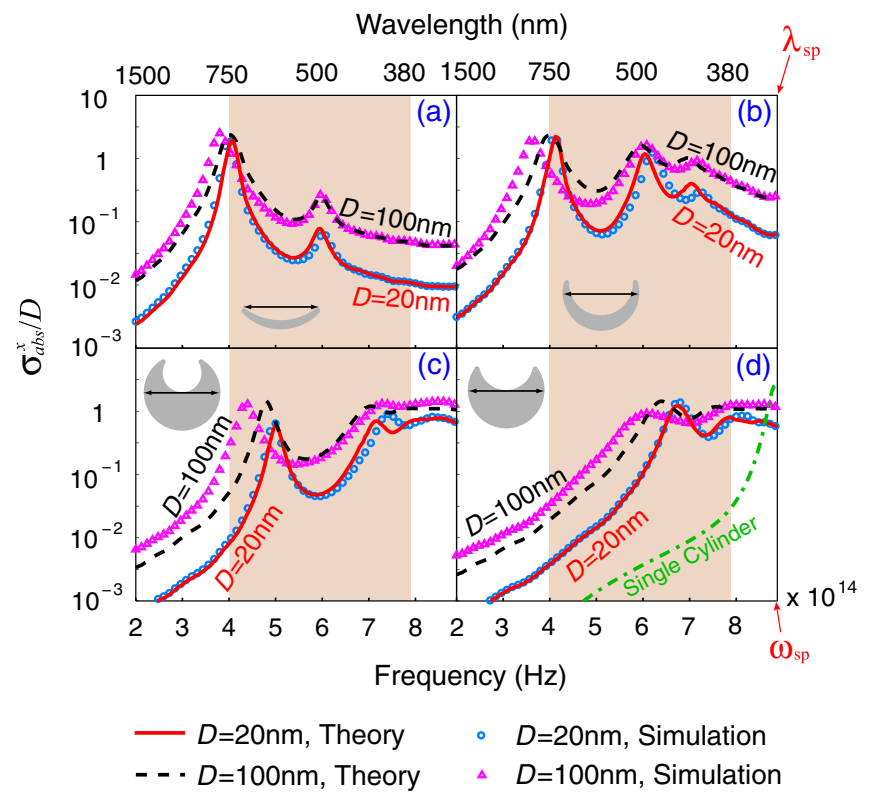

FIG. 3 (color online). Normalized absorption cross sections $\sigma_{\text {abs }}^{x} / D$ for blunt crescents of different shapes: (a) $\theta=9^{\circ}$ and $t=0.04 D$, (b) $\theta=9^{\circ}$ and $t=0.16 D$, (c) $\theta=9^{\circ}$ and $t=0.5 D$, and (d) $\theta=45^{\circ}$ and $t=0.5 D$. In each case, the bluntness dimension is fixed as $\delta_{1}=\delta_{2}=0.01 D$, and crescents of two different sizes $(D=20 \mathrm{~nm}$ and $D=100 \mathrm{~nm})$ are considered. The shaded (brown) regions in the subfigures identify the visible spectrum. Theoretical calculations are compared with numerical simulations.
Figure 3 shows the absorption spectrum (under $x$ polarization) for four blunt crescents with different shapes (different $t$ and $\theta$ ) but the same bluntness dimension $\left(\delta_{1}=\delta_{2}=0.01 D\right)$. In each case, crescents of two different sizes $(D=20 \mathrm{~nm}$ and $D=100 \mathrm{~nm})$ are considered. (Note that when $D>40 \mathrm{~nm}$, the radiative damping is no longer negligible. In our calculations, it is modeled as a fictive absorbing dipole in the initial space [19].) Numerical simulations performed using the software COMSOL are compared with the analytical calculations, and the good agreement demonstrates the validity of our analytical model (see the Supplemental Material [19] for more comparisons on different bluntness dimensions). It is worth pointing out that, for structures with larger physical sizes (e.g., $D=100 \mathrm{~nm}$ ), the numerical results are slightly redshifted from our theoretical predictions, which is a result of ignoring retardation effects in the analytical treatment.

Figure 3 demonstrates the strong dependence of SP excitations on crescent shape. Firstly, we find that the energy of higher-order modes is directly related to the crescent thickness $t$. For a thin flat crescent shown in Fig. 3(a) where $t=0.04 D$, only the dipole mode couples strongly to the incident light and all the higher-order modes are absent or weakly excited. As the crescent thickness increases to $t=0.16 D$ (while $\theta$ is kept unchanged), two high-order modes (with angular moment $n=3$ and 5) are excited in turn [see Fig. 3(b)]. When $t$ further increases to $0.5 \mathrm{D}$, all the higher-order modes emerge consecutively [see Fig. 3(c)]. The second point that can be observed is that the tip angle $\theta$ determines the linewidth and resonant frequencies of the SP modes: from Fig. 3(c) to Fig. 3(d), we keep $t$ unchanged while increasing $\theta$ to $45^{\circ}$. The 


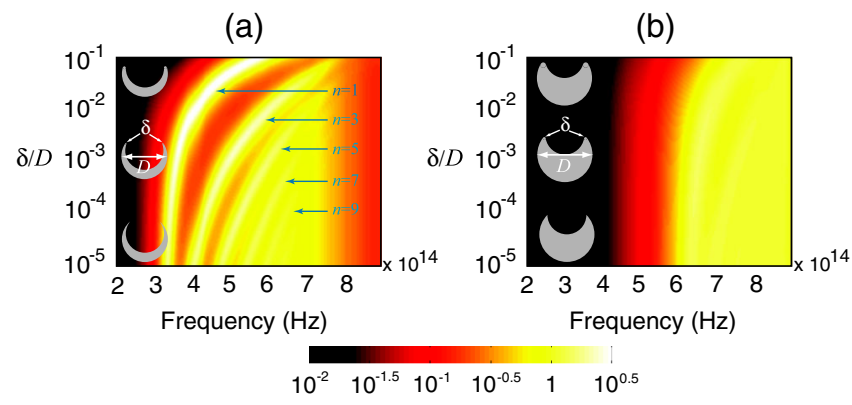

FIG. 4 (color online). Normalized absorption cross sections $\sigma_{\text {abs }}^{x} / D$ in terms of the frequency and $\delta / D$ : (a) the case for the blunt crescent taking the shape in Fig. 3(b) and (b) the case for the blunt crescent depicted in Fig. 3(d). Note that SP modes with even angular moment $n$ are absent in this case since the crescent takes a symmetric shape.

hybridization of SPs at the crescent tips results in a blueshift and increased linewidths of all the modes (especially for the lower-order ones), yielding a spread range of each resonance. Finally, all the resonance bands overlap with each other, giving rise to a continuous spectrum in the visible frequency range. The absorption cross section of a single cylinder is also plotted as a comparison to demonstrate the broadband light harvesting property of the crescent.

To further determine how the geometry bluntness affects the absorption behavior of the crescent, we calculate $\sigma_{\text {abs }}^{x} / D$ while varying the bluntness dimension $\delta_{1(2)}$. The absorption cross section is first calculated for a thin crescent with a small tip angle [here, we use the same geometry as shown in Fig. 3(b)]. As the bluntness dimension increases, all the resonances are blueshifted and finally degenerate at the surface plasmon frequency, as shown in Fig. 4(a). Nevertheless, for the crescent geometry in Fig. 3(d), the continuous absorption spectrum is nearly independent of the bluntness dimension until $\delta$ reaches up to $0.04 D$. This surprising result evidences the possibility of designing broadband light harvesting devices robust to the geometry bluntness.

In general, both the spectral position of the crescent and the local field enhancement depend upon the geometry bluntness. Therefore, in Fig. 5, we compare the normalized electric fields $E_{x}^{\prime} / E_{0}^{\prime}$ along the inner boundaries of the blunt (red dashed line) and the corresponding singular (blue solid line) crescents. As expected, the maximum field enhancement is distinctly decreased from the order of $10^{3}$ to 25 as the claw tips are turned blunt. However, away from the tip, the field only undergoes minor changes due to the bluntness. It should be pointed out that quantum mechanical effects are not considered in our calculation. For subnanometer bluntness dimensions, a more general quantum description and nonlocal constitutive relation of the metal may be necessary to further improve our analytical model $[21,22]$.
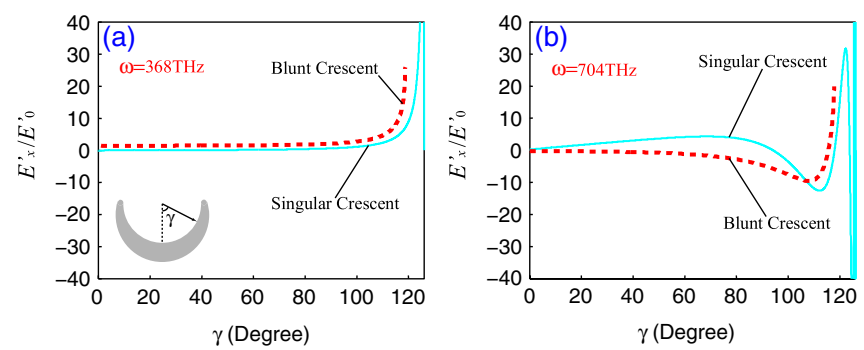

FIG. 5 (color online). Normalized electric fields $E_{x}^{\prime} / E_{0}^{\prime}$ along the inner boundaries of the blunt and the corresponding singular crescents at two different frequencies: (a) $368 \mathrm{THz}$ (the resonance of the dipole mode) and (b) $704 \mathrm{THz}$ (the resonance of a higher-order mode). Here, the geometry parameters are set as $\theta=9^{\circ}, t=0.16 D$, and $\delta_{1}=\delta_{2}=0.01 D$ (for the blunt crescent). Because of the limitation of space, the curves for the singular cases are truncated in the vertical axis of the plots.

To conclude, this Letter describes a systematic methodology to address the dependence of resonance properties and field enhancement of SP modes on the geometry of blunt nanostructures. Our analytical study on 2D nanocrescents provides a unique understanding of the localized SP modes and reveals the physics behind those phenomena. Because of its ease of applicability, we anticipate that this approach will be of value in understanding and predicting the effects of edge rounding in a variety of plasmonic structures, such as wedges [9-12], overlapping cylinders [23], and rough surfaces [24,25]. In conjunction with a recent 3D transformation optics approach [26], our analytical model may even be generalized to treat 3D plasmonic structures with blunt tips. For example, a kissing spherical dimer with a blunt touching point can be mapped to a cylindrical plasmonic cavity, where the surface plasmon excitations may be analytically described. Extended study on this topic will be performed in our future work. Unlike a numerical method, the analytical strategy considered here allows for an efficient and rapid optimization of the nanostructures, which not only opens the possibility of designing a bluntness-independent wideband light harvesting device but also has implications for numerous optical applications, such as nonlinear phenomena and molecular spectroscopy.

This work is supported by the European Community project PHOME (Contract No. 213390), by the U.K. Engineering and Physical Sciences Research Council (EPSRC), and by the Leverhulme Trust.

*y.luo09@imperial.ac.uk

[1] S. A. Maier and H. A. Atwater, J. Appl. Phys. 98, 011101 (2005).

[2] N. J. Halas, S. Lai, W. S. Chang, S. Link, and P. Nordlander, Chem. Rev. 111, 3913 (2011).

[3] W. L. Barnes, A. Dereux, and T.W. Ebbesen, Nature (London) 424, 824 (2003). 
[4] D. K. Gramotnev and S. I. Bozhevolnyi, Nature Photon. 4, 83 (2010).

[5] A. Aubry, D. Y. Lei, A. I. Fernandez-Dominguez, Y. Sonnefraud, S.A. Maier, and J.B. Pendry, Nano Lett. 10, 2574 (2010).

[6] Y. Luo, J. B. Pendry, and A. Aubry, Nano Lett. 10, 4186 (2010).

[7] J. B. Pendry, D. Schurig, and D. R. Smith, Science 312, 1780 (2006).

[8] A. Aubry, D. Y. Lei, S. A. Maier, and J. B. Pendry, Phys. Rev. B 82, 205109 (2010).

[9] E. Moreno, S. G. Rodrigo, S. I. Bozhevolnyi, L. MartínMoreno, and F. J. García-Vidal, Phys. Rev. Lett. 100, 023901 (2008).

[10] D. F. P. Pile, T. Ogawa1, D. K. Gramotnev, T. Okamoto, M. Haraguchi, M. Fukui, and S. Matsuo, Appl. Phys. Lett. 87, 061106 (2005).

[11] H. Choi, D. F. Pile, S. Nam, G. Bartal, and X. Zhang, Opt. Express 17, 7519 (2009).

[12] J. Zhang, S. Xiao, M. Wubs, and N. A. Mortensen, ACS Nano 5, 4359 (2011).

[13] J. Kim, G. Liu, Y. Lu, and L. Lee, Opt. Express 13, 8332 (2005).

[14] B. M. Ross and L.P. Lee, Nanotechnology 19, 275201 (2008).
[15] L. Y. Wu, B. M. Ross, and L. P. Lee, Nano Lett. 9, 1956 (2009).

[16] J. S. Shumaker-Parry, H. Rochholz, and M. Kreiter, Adv. Mater. 17, 2131 (2005).

[17] H. Rochholz, N. Bocchlo, and M. Kreiter, New J. Phys. 9, 53 (2007).

[18] R. Bukasov and J. S. Shumaker-Parry, Nano Lett. 7, 1113 (2007).

[19] See Supplemental Material at http://link.aps.org/ supplemental/10.1103/PhysRevLett.108.023901 for detailed derivations on periodic truncated metal slabs.

[20] E. D. Palik, Handbook of Optical Constants of Solids (Academic, New York, 1991), Vol. II.

[21] J. Zuloaga, E. Prodan, and P. Nordlander, Nano Lett. 9, 887 (2009).

[22] J. Lindhardt, K. Dan. Vidensk. Selsk. Mat. Fys. Medd. 28, 8 (1954).

[23] D. Y. Lei, A. Aubry, Y. Luo, S. A. Maier, and J. B. Pendry, ACS Nano 5, 597 (2011).

[24] F. J. García-Vidal and J. B. Pendry, Phys. Rev. Lett. 77, 1163 (1996).

[25] Y. Luo, A. Aubry, and J.B. Pendry, Phys. Rev. B 83, 155422 (2011).

[26] A. I. Fernández-Domínguez, S. A. Maier, and J. B. Pendry, Phys. Rev. Lett. 105, 266807 (2010). 